\title{
Specify Therapeutic Agent
}

National Cancer Institute

\section{Source}

National Cancer Institute. Specify Therapeutic Agent. NCI Thesaurus. Code C157096.

A directive to record the therapeutic agent or agents. 